\title{
Current Status of Radiologic Diagnosis for Mediastinal Lymph Node Metastases of Non-Small-Cell Lung Cancer: Retrospective Study of pN2 Cases
}

\author{
Shinsuke Saisho", Koichiro Yasuda, Ai Maeda, Takuro Yukawa, Riki Okita, Yuji Hirami, \\ Katsuhiko Shimizu, Masao Nakata \\ Department of General Thoracic Surgery, Kawasaki Medical School Hospital, Kurashiki, Japan. \\ Email: *s.saisho@med.kawasaki-m.ac.jp
}

Received August 24 $4^{\text {th }}, 2012$; revised September $24^{\text {th }}, 2012$; accepted October $10^{\text {th }}, 2012$

\begin{abstract}
Objective: Advances in diagnostic imaging techniques, such as ${ }^{18} \mathrm{~F}$-fluorodeoxyglucose positron emission tomography (FDG-PET), have led to greater accuracy in preoperative mediastinal staging for patients with non-small-cell lung cancer (NSCLC), but surgical staging remains the "gold standard" for diagnosis. A proper understanding of the current accuracy of diagnostic imaging is needed for further improvements. Methods: Forty-three patients who underwent resection for NSCLC involving mediastinal lymph node (MLN) metastasis at our hospital between June 2003 and May 2011 were enrolled in this study. We conducted a retrospective study of the radiological and pathological findings for 53 metastatic MLNs in the 43 patients. Results: The preoperative imaging modality was computed tomography (CT) alone for 18 patients (22 MLNs) and CT and FDG-PET for 25 patients (31 MLNs). The sensitivities of CT and FDG-PET were $41.5 \%$ and $58.0 \%$, respectively. The sensitivity of CT did not differ according to any clinicopathological factors, but the sensitivity of FDG-PET tended to be higher for primary tumors with high SUV $_{\max }$ values and for non-adenocarcinomas. In the lymph nodes, all micrometastatic foci $\leq 2 \mathrm{~mm}$ were PET-negative, but 4 lymph nodes with metastatic foci larger than $10 \mathrm{~mm}$ were also PET-negative. Conclusions: For the diagnostic imaging of MLN, FDG-PET has a greater sensitivity than contrast-enhanced CT based on "size criteria", but it is still not sufficiently sensitive and is influenced by various factors. At present, histological confirmation of MLNs is necessary when making decisions regarding treatment plans and the type of surgical procedure that should be performed.
\end{abstract}

Keywords: Non-Small-Cell Lung Cancer; Mediastinal Lymph Node Metastasis; Positron Emission Tomography; Computed Tomography

\section{Introduction}

Mediastinal lymph node (MLN) metastasis is the most important factor in determining both the treatment strategy and the prognosis of patients with non-small-cell lung cancer (NSCLC) without distant metastasis [1,2]. Several non-invasive and invasive diagnostic procedures have been used for mediastinal staging before surgery with curative intent, including computed tomography (CT), ${ }^{18}$ F-fluorodeoxyglucose positron emission tomography (FDG-PET), and endobronchial ultrasound with transbronchial needle aspiration (EBUS-TBNA), mediastinoscopy, and video-assisted thoracoscopy (VATS). Non-invasive radiographic staging with contrast-enhanced $\mathrm{CT}$ and FDG-PET remain the standard procedures. However, neither CT nor FDG-PET is sufficiently sensitive or specific for the diagnosis of MLN metastasis [3-6]. The

\footnotetext{
"Corresponding author.
}

American College of Chest Physicians (ACCP) guidelines and the European Society of Thoracic Surgeons (ESTS) guidelines state: "in patients with discrete lymph node enlargement, staging by CT or FDG-PET is not sufficiently accurate, and invasive surgical procedures are recommended." [7-9].

In the current study, we investigated the diagnostic significance of CT and FDG-PET for identifying MLN metastases and compared the findings obtained using these imaging modalities with the histological results.

\section{Patients and Methods}

\subsection{Study Population}

Of the 53 patients who underwent surgery and were diagnosed as having pN2 NSCLC at the Kawasaki Medical School Hospital, Kurashiki, Japan, between June 2003 and May 2011, we included 43 patients who had under- 
gone contrast-enhanced CT before surgery in the current study. Ten patients who underwent only plain CT preoperatively were excluded.

A total of 53 metastatic MLNs were evaluated in the 43 patients. The histological type was adenocarcinoma in 24 patients, squamous cell carcinoma in 12, large cell carcinoma in 3 , adenosquamous carcinoma in 2 , and pleomorphic carcinoma in 2. Localization of the primary tumor was peripheral-type in 39 patients and central-type in 4 . The pathological N2 status was single station in 34 patients and multiple stations in 9 (Table 1). The lymph node station was determined according to the lymph node map proposed by the International Association for the Study of Lung Cancer (IASLC). Written informed consent was obtained from each patient, and the study was approved by the institutional review board of Kawasaki Medical School and Hospital (IRB No. 967).

\subsection{CT Imaging}

The CT scans were performed using a 16-detector-row device (LightSpeed 16; GE Healthcare, Milwaukee, WI), and the scanning parameters were as follows: exposure settings, 323 to $432 \mathrm{~mA}, 0.5 \mathrm{~s} /$ rotation at $120 \mathrm{kVp}$; collimation, $16 \times 0.625$ or $1.25 \mathrm{~mm}$; beam pitch, 1.75:1. A 10 -mm-thick contiguous collimation was used to evaluate the entire lung for the preoperative evaluations. The size of the tumors and MLNs were determined digitally based on the findings of a thin-section CT scan.

For each metastatic MLN station, the short-axis diameter of the largest lymph node was measured using CT. The "size criteria" of the CT definition for MLN metastasis was a short-axis diameter of $10 \mathrm{~mm}$ or larger.

\subsection{FDG-PET Imaging}

FDG-PET was performed using a dedicated PET/CT scanner (Discovery ST Elite; GE Healthcare, Kyoto, Japan). The axes of the multidetector CT and PET systems were mechanically aligned so that the patient could be moved from the CT to the PET scanner gantry by simply changing the position of the examination table. The resultant PET and CT scans were coregistered with hardware. PET/CT scanning was performed at 115 minutes after the intravenous injection of 150 to $220 \mathrm{MBq}$ of 18-fluorodeoxyglucose (FDG scan; Universal Giken, Nihon Medi-Physics, Tokyo, Japan). The regions of interest were placed three-dimensionally over the lung cancer nodules. A semiquantitative analysis of the images was performed by measuring the maximal standardized uptake value $\left(\mathrm{SUV}_{\max }\right)$ of the lesions. The SUV was calculated using the following equation: tumor activity concentration/(injected dose/body weight).

The FDG-PET criteria for MLN metastasis were de-
Table 1. Patients' characteristics.

\begin{tabular}{|c|c|c|}
\hline & & $\mathrm{n}=43$ \\
\hline \multirow[t]{2}{*}{ Age (years) } & Median & 71 \\
\hline & Range & $45-83$ \\
\hline \multirow[t]{2}{*}{ Sex } & Male & 27 \\
\hline & Female & 16 \\
\hline \multirow[t]{3}{*}{ Smoking Index (pack-years) } & 0 & 17 \\
\hline & $1-20$ & 7 \\
\hline & $>20$ & 19 \\
\hline \multirow[t]{3}{*}{ Tumor Marker (CEA) } & $\leq 5.0 \mathrm{ng} / \mathrm{mL}$ & 19 \\
\hline & $>5.0 \mathrm{ng} / \mathrm{mL}$ & 23 \\
\hline & unknown & 1 \\
\hline \multirow[t]{2}{*}{ Tumor Location } & Central & 4 \\
\hline & Peripheral & 39 \\
\hline \multirow[t]{2}{*}{ Tumor Size $(\mathrm{cm})$} & Average & 3.2 \\
\hline & Range & $1.2-6.6$ \\
\hline \multirow[t]{5}{*}{ Histology } & Adenocarcinoma & 24 \\
\hline & Squamous cell carcinoma & 12 \\
\hline & Large cell carcinoma & 3 \\
\hline & Adenosquamous carcinoma & 2 \\
\hline & Pleomorphic carcinoma & 2 \\
\hline \multirow[t]{2}{*}{ pN1 Status } & pN1 (-) & 11 \\
\hline & pN1 (+) & 32 \\
\hline \multirow[t]{2}{*}{ pN2 Status } & $\mathrm{N} 2$ single station & 34 \\
\hline & N2 multiple station & 9 \\
\hline
\end{tabular}

CEA: carcinoembryonic antigen.

fined as FDG uptake coinciding with MLNs irrespective of the $\mathrm{SUV}_{\max }$ and exhibiting a left-right asymmetry.

\subsection{Microscopic Measurement of the Size of the Lymph Nodes and Metastatic Foci}

The dissected lymph nodes were histologically examined using $10 \%$ formalin-fixed and paraffin-embedded sections with hematoxylin and eosin staining. In each lymph node containing metastases, the lymph node with the largest metastatic foci was selected, and the long-axis and short-axis diameters of the metastatic foci in the lymph nodes were microscopically measured.

\subsection{Statistical Analysis}

The clinical and pathological parameters were compared using a chi-square test. Univariate analyses were performed 
using the log-rank test. Differences were considered significant if $P \leq 0.05$. The statistical analyses were performed using a statistical software package (SPSS).

\section{Results}

\subsection{CT and FDG-PET Findings}

The characteristics and radiologic findings of 53 metastatic MLNs are shown in Table 2. Of 53 metastatic MLNs, 49 lymph nodes were visible on preoperative CT and the remaining 4 were not. The lymph node short-axis diameters were $4.7-25.1 \mathrm{~mm}$ (median, $9.6 \mathrm{~mm}$ ) and were $\geq 10 \mathrm{~mm}$ in 22 lymph nodes. The sensitivity of CT was $41.5 \%$.

FDG-PET was performed in 26 of 43 patients, with a total of 31 MLNs imaged. Eighteen lymph nodes showed FDG uptake (13 nodes with $\mathrm{SUV}_{\max } \geq 2.5 ; 5$ nodes with $\mathrm{SUV}_{\max }<2.5$ ), and the remaining 13 nodes showed no FDG uptake. The sensitivity of FDG-PET was $58.0 \%$.

When defining either CT or FDG-PET findings as positive for lymph node metastasis (cN2), diagnostic imaging combining CT and FDG-PET had a diagnostic sensitivity of $67.7 \%$.

\subsection{Correlation between CT/FDG-PET Findings and Clinicopathological Factors}

We investigated the clinicopathological factors influencing the sensitivity of CT and FDG-PET (Table 3). We did not find any factors influencing the sensitivity of CT for the diagnosis of MLN metastasis. In contrast, the sensitivity of FDG-PET for the diagnosis of MLN metastasis was significantly higher $(P=0.001)$ when the primary tumor $\mathrm{SUV}_{\max }$ was $>10.0$. Also, in terms of histology, the sensitivity tended to be higher for non-adenocarcinoma than for adenocarcinoma $(P=0.075)$.

\subsection{Size of Metastatic Foci in Lymph Nodes}

The size of the metastatic foci could be measured pathologically in 44 metastatic MLNs. These foci were

Table 2. Characteristics and radiologic findings of 53 metastatic mediastinal lymph nodes.

\begin{tabular}{|c|c|c|c|c|c|}
\hline & & $\mathrm{N}$ & \multicolumn{3}{|c|}{ Detail } \\
\hline \multirow{4}{*}{ pN2 Station } & Upper Zone & 26 & \multicolumn{3}{|c|}{$\# 2 \mathrm{R}(\mathrm{n}=1), \# 3 \mathrm{a}(\mathrm{n}=2), \# 4 \mathrm{~L}(\mathrm{n}=3), \# 4 \mathrm{R}(\mathrm{n}=20)$} \\
\hline & AP Zone & 7 & \multicolumn{3}{|c|}{$\# 5(n=6), \# 6(n=1)$} \\
\hline & Subcarinal Zone & 17 & \multicolumn{3}{|c|}{$\# 7(\mathrm{n}=17)$} \\
\hline & Lower Zone & 3 & \multicolumn{3}{|c|}{$\# 8 \mathrm{~L}(\mathrm{n}=1), \# 8 \mathrm{R}(\mathrm{n}=1), \# 9 \mathrm{~L}(\mathrm{n}=1)$} \\
\hline \multirow[t]{2}{*}{ Histology } & Adenocarcinoma & 33 & & & \\
\hline & Non-Adenocarcinoma & 20 & & & \\
\hline \multirow[t]{5}{*}{ CT Findings } & Detected & 49 & Short-Axis (mm) & Average & $10.0 \mathrm{~mm}$ \\
\hline & & & & Range & $4.7-25.1 \mathrm{~mm}$ \\
\hline & & & & $>10 \mathrm{~mm}$ & $\mathrm{n}=22$ \\
\hline & & & & $\leq 10 \mathrm{~mm}$ & $\mathrm{n}=27$ \\
\hline & Not Detected & 4 & & & \\
\hline \multirow[t]{5}{*}{ PET Findings } & FDG Uptake $(+)$ & 18 & $\mathrm{SUV}_{\max }$ & Average & 5.2 \\
\hline & & & & Range & $1.0-12.1$ \\
\hline & & & & $>2.5$ & $\mathrm{n}=13$ \\
\hline & & & & $\leq 2.5$ & $\mathrm{n}=5$ \\
\hline & FDG Uptake (-) & 13 & & & \\
\hline \multirow[t]{4}{*}{$\mathrm{CT}$ and PET } & $\mathrm{CT}(+)^{*} / \mathrm{PET}(+)^{* *}$ & 7 & & & \\
\hline & $\mathrm{CT}(+) / \operatorname{PET}(-)$ & 3 & & & \\
\hline & $\mathrm{CT}(-) / \operatorname{PET}(+)$ & 11 & & & \\
\hline & $\mathrm{CT}(-) / \operatorname{PET}(-)$ & 10 & & & \\
\hline
\end{tabular}

CT: computed tomography; PET: positron emission tomography; FDG: 18 F-fluorodeoxyglucose; SUV $\max$ : the maximal standardized uptake value; ${ }^{*} \mathrm{CT}(+)$ : short-axis diameter of mediastinal lymph node $\geq 10 \mathrm{~mm} ;{ }^{* *}$ PET $(+)$ : presence of asymmetrical FDG uptake coincided with mediastinal lymph node. 
Table 3. Correlations with clinicopathological factors in 53 metastatic mediastinal lymph nodes.

\begin{tabular}{|c|c|c|c|c|c|}
\hline & & \multicolumn{2}{|c|}{ CT Findings } & \multicolumn{2}{|c|}{ PET Findings } \\
\hline & & Sensitivity (\%) & $P$ & Sensitivity (\%) & $P$ \\
\hline \multirow[t]{2}{*}{ Age } & $\geq 70 \mathrm{yr}$ & 47 & 0.275 & 73 & 0.055 \\
\hline & $<70 \mathrm{yr}$ & 71 & & 47 & \\
\hline \multirow[t]{2}{*}{ Sex } & Male & 60 & 0.882 & 45 & 0.178 \\
\hline & Female & 57 & & 89 & \\
\hline \multirow[t]{2}{*}{ Smoking Status } & Smoker & 58 & 0.933 & 50 & 0.162 \\
\hline & Non-Smoker & 60 & & 77 & \\
\hline \multirow[t]{2}{*}{ CEA } & $>5.0 \mathrm{ng} / \mathrm{mL}$ & 60 & 0.949 & 52 & 0.340 \\
\hline & $\leq 5.0 \mathrm{ng} / \mathrm{mL}$ & 48 & & 66 & \\
\hline \multirow[t]{2}{*}{ Histology } & Adenocarcinoma & 64 & 0.293 & 44 & 0.075 \\
\hline & Non-Adenocarcioma & 50 & & 76 & \\
\hline \multirow[t]{2}{*}{ Location } & Central & 75 & 0.509 & 100 & 0.222 \\
\hline & Peripheral & 58 & & 55 & \\
\hline \multicolumn{6}{|l|}{ Primary Tumor } \\
\hline \multirow[t]{2}{*}{ Size } & $>3.0 \mathrm{~cm}$ & 55 & 0.514 & 68 & 0.221 \\
\hline & $\leq 3.0 \mathrm{~cm}$ & 64 & & 46 & \\
\hline \multirow[t]{2}{*}{$\mathrm{SUV}_{\max }$} & $>10.0$ & 62 & 0.526 & 87 & 0.001 \\
\hline & $\leq 10.0$ & 73 & & 26 & \\
\hline \multirow[t]{2}{*}{ pN1 Status } & pN1 (+) & 59 & 0.965 & 57 & 0.882 \\
\hline & pN1 (-) & 58 & & 60 & \\
\hline \multirow[t]{3}{*}{ pN2 Status } & Superior & 63 & 0.415 & 59 & 0.790 \\
\hline & Aortic & 71 & & 67 & \\
\hline & Inferior & 50 & & 50 & \\
\hline
\end{tabular}

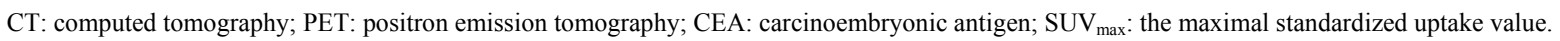

significantly larger $(P=0.001)$ in CT-positive lymph nodes with a diameter of $10 \mathrm{~mm}$ or more. In contrast, no statistical correlation was observed between the size of the metastatic foci and the FDG uptake (Figures 1 and 2).

\subsection{False-Negative Lymph Nodes with FDG-PET}

Thirteen lymph nodes from 9 patients showed no FDG uptake, as summarized in Table 4. In 10 of these 13 nodes, the histology was adenocarcinoma. The metastatic foci were micrometastatic lesions $\leq 2 \mathrm{~mm}$ in 7 of 13 nodes. On the other hand, in 4 of the lymph nodes, the metastatic focus showed no FDG uptake, despite having a long axis greater than $10 \mathrm{~mm}$.

\section{Discussion}

MLN metastasis is the most important factor when de- termining the treatment plan for resectable NSCLC. Recent advances in diagnostic imaging technology, particularly the introduction of FDG-PET, have enabled more accurate reoperative diagnoses. In meta-analyses of reports describing the diagnosis of lymph node metastasis during the years 2000-2009, FDG-PET demonstrated a better diagnostic rate than CT, with a sensitivity of $70 \%$ $85 \%$ and a specificity of $90 \%-95 \%$, compared to a sensitivity of $60 \%$ and a specificity of $75 \%-80 \%$ for CT. [3-6] However, the accuracy of FDG-PET differed according to various factors, such as histology[10], lymph node station [11], size of lymph nodes [12,13], size of metastatic foci inside lymph nodes [13,14], and the localization of primary tumor [12]. Moreover, $\mathrm{SUV}_{\max }$ was affected by various factors including the equipment that was used, the imaging conditions, image reconstruction, and the patient's condition; thus, comparisons among 
Table 4. Pathological findings of 13 lymph nodes with false-negative of FDG-PET results.

\begin{tabular}{|c|c|c|c|c|c|c|c|}
\hline \multirow[b]{2}{*}{ Case } & \multirow[b]{2}{*}{ Histology } & \multicolumn{3}{|c|}{ Primary Tumor } & \multicolumn{3}{|c|}{ pN2 Nodes } \\
\hline & & Location & Size (mm) & $\mathrm{SUV}_{\max }$ & Station & Size $(\mathrm{mm})$ & $\begin{array}{l}\text { Microscopic Size of } \\
\text { Metastatic Foci (mm) }\end{array}$ \\
\hline 1 & Ad & RU & 31 & 10.8 & $\# 4 \mathrm{R}$ & $(-)$ & $<1 \times 1$ \\
\hline \multirow[t]{2}{*}{2} & Ad & RL & 45 & 7 & $\# 4 \mathrm{R}$ & 7.6 & $<1 \times 1$ \\
\hline & & & & & $\# 7$ & 10.0 & $<1 \times 1$ \\
\hline 3 & $\mathrm{Ad}$ & LU & 30 & 4.1 & $\# 6$ & 6.8 & $<1 \times 1$ \\
\hline \multirow[t]{2}{*}{4} & $\mathrm{Ad}$ & LU & 25 & 5.3 & $\# 4 \mathrm{~L}$ & 8.2 & $<1 \times 1$ \\
\hline & & & & & $\# 5$ & $(-)$ & $<1 \times 1$ \\
\hline 5 & $\mathrm{AdSq}$ & RL & 22 & 5.2 & $\# 7$ & 8.5 & $6 \times 3$ \\
\hline \multirow[t]{2}{*}{6} & $\mathrm{Ad}$ & RU & 18 & 4.5 & $\# 2 \mathrm{R}$ & 9.6 & $2 \times 1$ \\
\hline & & & & & $\# 4 \mathrm{R}$ & 12.0 & $10 \times 8$ \\
\hline 7 & $\mathrm{Sq}$ & $\mathrm{RL}$ & 25 & 8.8 & $\# 3 \mathrm{a}$ & 9.9 & $12 \times 11$ \\
\hline 8 & $\mathrm{Sq}$ & LL & 25 & 10.6 & $\# 7$ & 4.8 & $12 \times 11$ \\
\hline \multirow[t]{2}{*}{9} & $\mathrm{Ad}$ & $\mathrm{RM}$ & 31 & 7.6 & $\# 4 \mathrm{R}$ & 10.5 & $28 \times 14$ \\
\hline & & & & & $\# 7$ & 8.2 & $5 \times 4$ \\
\hline
\end{tabular}

Ad: adenocarcinoma, AdSq: adenosquamous carcinoma. Sq: squzmous cell carcinoma, RU: rught upper lobe, RM: right middle lobe, RL: right lower lobe, LU: left upper lobe, LL: left lower lobe.

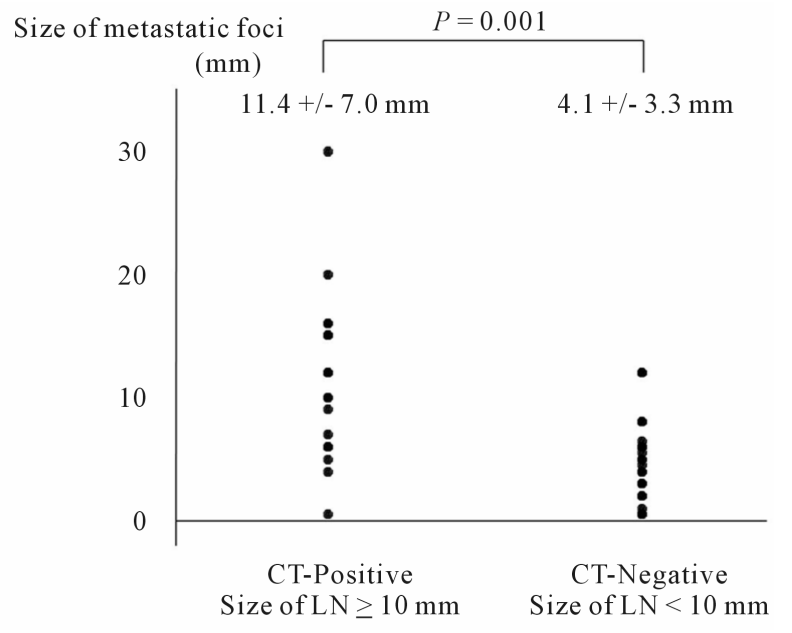

Figure 1. Distribution of sizes of metastatic foci in CT-positive (short-axis $\geq 10 \mathrm{~mm}$ ) and CT-negative (short-axis $<10$ mm) lymph nodes as observed using CT.

multiple institutions are problematic. For these reasons, no clear diagnostic "criteria" have been established for FDG-PET diagnosis of MLN metastasis. In our study, when we adopted 1) a clear FDG uptake compared with the area around the mediastinal tissue and 2) a left-right asymmetry as the criteria for positive metastasis, the sensitivity was $58 \%$, which was similar to the results of previous reports. We also observed differences in FDG-PET

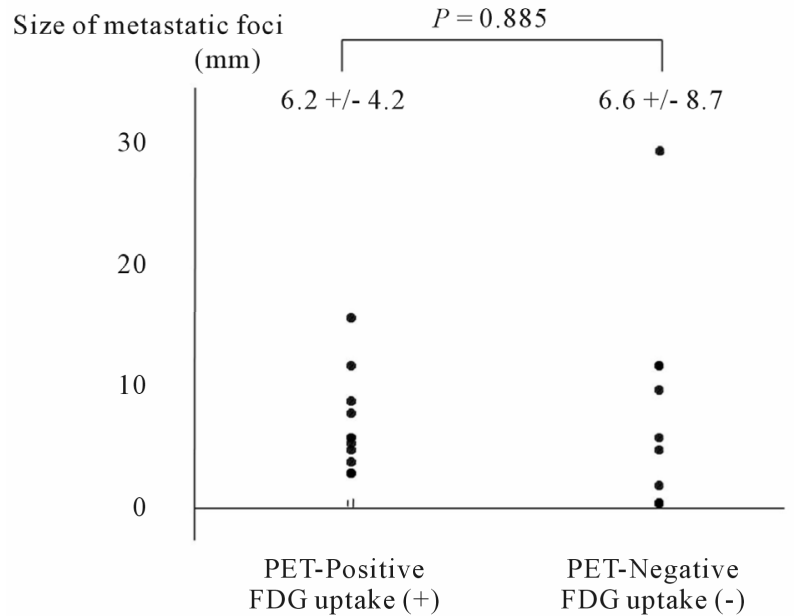

Figure 2. Distribution of sizes of metastatic foci in PETpositive and PET-negative lymph nodes as observed using FDG-PET.

sensitivity according to histology (44\% for adenocarcinoma versus $77 \%$ for non-adenocarcinoma, $P=0.075$ ) and $\mathrm{SUV}_{\max }$ of primary tumor $(26 \%$ for tumors with $\mathrm{SUV}_{\max } \leq 10$ versus $87 \%$ for tumors with $\mathrm{SUV}_{\max }>10, P$ $=0.001$ ); however, the sensitivity did not differ according to the localization of primary tumors (central-type or peripheral-type), the metastatic lymph node station, or the presence of hilar lymph node metastases. These re- 
sults demonstrate that when judging the presence of MLN metastasis using FDG-PET diagnostic images, various pathological factors must be taken into account in addition to the lymph node size and $\mathrm{SUV}_{\max }$. Thus, we believe that it may be difficult to establish "cN2 criteria" for FDG-PET imaging.

Using FDG-PET, the underestimation of the $\mathrm{SUV}_{\max }$ in small pulmonary lesions has been reported, and this issue is an important problem in the diagnosis of lymph node metastasis. The sizes of the metastatic foci in lymph nodes influence the diagnostic ability, and FDG-PET is relatively poor at identifying metastatic foci of $4-10 \mathrm{~mm}$ in size $[13,14]$. In our study, all the lymph node metastatic foci with FDG uptake were $\geq 3 \mathrm{~mm}$ in size, and the absence of FDG uptake occurred in micrometastatic lesions $\leq 2 \mathrm{~mm}$ in size. This low ability to detect small metastatic foci is a limitation of FDG-PET. On the other hand, we unexpectedly observed 4 lymph nodes that were PET-negative despite being at least $10 \mathrm{~mm}$ in size and having metastatic lesions distributed throughout the whole lymph node. Such lymph nodes would have been assessed as "CT-positive, PET-negative" in clinical practice and would likely have been judged as reactive lymph node swelling (cN0).

EBUS-TNBA is a new, minimally invasive method for histologically confirming metastatic MLNs. However, biopsies are only performed for lymph nodes with suspected metastases based on CT or FDG-PET findings. In addition, not all MLNs are approachable using EBUSTNBA or a mediastinoscopy. Thus, diagnostic imaging still plays a major role in preoperative staging, yet the diagnostic accuracy of these techniques has not improved. In recent years, induction therapy followed by surgery has become more common for the treatment of resectable cN2 NSCLC. We are also seeing a growing diversity of therapeutic and surgical procedures at different clinical stages, e.g., a simplified lymphadenectomy, such as a lobe-selective lymph node dissection, and limited resections for small-sized NSCLC. Preoperative diagnostic imaging is therefore becoming even more important. A careful approach conducted with an understanding of the current state of diagnostic imaging and its uncertainties and with the application of EBUS-TBNA or mediastinoscopy, when necessary, in addition to pathological examinations, such as intraoperative biopsy, is required.

Our study has some limitations. First, the numbers of patients and lymph nodes that were examined were relatively small, and only $60 \%$ of all the lymph nodes were imaged using FDG-PET. Second, we only studied lymph nodes that were confirmed as being histologically metastatic. A study of the CT and FDG-PET findings for a larger number of patients and lymph nodes, including non-metastatic lymph nodes, is needed.

\section{Conclusion}

The sensitivity of diagnostic imaging for MLN metastasis was $41.5 \%$ for contrast-enhanced CT and $58.0 \%$ for FDG-PET. These sensitivities equate to a false-negative rate of over $30 \%$, even when CT and FDG-PET are used in combination. The accuracy of diagnostic imaging differs according to various factors, so histological confirmation is essential for choosing a treatment strategy and surgical procedures.

\section{REFERENCES}

[1] C. F. Mountain, "Revisions in the International System for Staging Lung Cancer," Chest, Vol. 111, No. 6, 1997 pp. 1710-1717. doi:10.1378/chest.111.6.1710

[2] J. LoCicero, "Surgical Treatment of Non-Small Cell Lung Cancer. In: T. W. Shields, J. LoCicero, B. P. Ronald and V. W. Rusch, Eds., General Thoracic Surgery, 7th Edition, Lippincott Williams \& Wilkins, Philadelphia, 2009, pp. 1388-1425.

[3] B. A. Dwamena, S. S. Sonnad, J. O. Angobaldo and R. L. Wahl, "Metastases from Non-Small Cell Lung Cancer: Mediastinal Staging in the 1990s-Meta-Analytic Comparison of PET and CT," Radiology, Vol. 213, No. 2, 1999, pp. 530-536.

[4] M. K. Gould, W. G. Kuschner, C. E. Rydzak, C. C. Maclean, A. N. Demas, H. Shigemitsu, et al., "Test Performance of Positron Emission Tomography and Computed Tomography for Mediastinal Staging in Patients with Non-Small-Cell Lung Cancer," Annals of Internal Medicine, Vol. 139, No. 11, 2003, pp. 879-892.

[5] O. Birim, A. P. Kappetein, T. Stijnen and A. J. Bogers, "Meta-Analysis of Positron Emission Tomographic and Computed Tomographic Imaging in Detecting Mediastinal Lymph Node Metastases in Non-Small Cell Lung Cancer," The Annals of Thoracic Surgery, Vol. 79, No. 1, 2005, pp. 375-381. doi:10.1016/j.athoracsur.2004.06.041

[6] Y. L. Lv, D. M. Yuan, K. Wang, X. H. Miao, Q. Qian, S. Z. Wei, et al., "Diagnostic Performance of Integrated Positron Emission Tomography/Computed Tomography for Mediastinal Lymph Node Staging in Non-Small Cell Lung Cancer: A Bivariate Systematic Review and MetaAnalysis," Journal of Thoracic Oncology, Vol. 6, No. 8, 2011, pp. 1350-1358. doi:10.1097/JTO.0b013e31821d4384

[7] G. A. Silvestri, M. K. Gould, M. L. Margolis, L. T. Tanoue, D. McCrory, E. Toloza, et al., "American College of Chest Physicians. Noninvasive Staging of NonSmall Cell Lung Cancer: ACCP Evidenced-Based Clinical Practice Guidelines (2nd Edition)," Chest, Vol. 132, Suppl. 3, 2007, pp. 178S-201S.

[8] F. C. Detterbeck, M. A. Jantz, M. Wallace, J. Vansteenkiste, G. A. Silvestri and American College of Chest Physicians, "Invasive Mediastinal Staging of Lung Cancer: ACCP Evidence-Based Clinical Practice Guidelines (2nd Edition)," Chest, Vol. 132, Suppl. 3, 2007, pp. 202S-220S.

[9] P. De Leyn, D. Lardinois, P. E. Van Schil, R. Rami-Porta, 
B. Passlick, M. Zielinski, et al., "ESTS Guidelines for Preoperative Lymph Node Staging for Non-Small Cell Lung Cancer," European Journal Cardio-Thoracic Surgery, Vol. 32, No. 1, 2007, pp. 1-8. doi:10.1016/j.ejcts.2007.01.075

[10] A. Billé, E. Pelosi, A. Skanjeti, V. Arena, L. Errico, P. Borasio, et al., "Preoperative Intrathoracic Lymph Node Staging in Patients with Non-Small-Cell Lung Cancer: Accuracy of Integrated Positron Emission Tomography and Computed Tomography," European Journal CardioThoracic Surgery, Vol. 36, No. 3, 2009, pp. 440-445. doi:10.1016/j.ejcts.2009.04.003

[11] R. J. Cerfolio, B. Ojha, A. S. Bryant, C. S. Bass, A. A. Bartalucci and J. M. Mountz, "The Role of FDG-PET Scan in Staging Patients with Non-Small Cell Carcinoma," The Annals of Thoracic Surgery, Vol. 76, No. 3, 2003, pp. 861-866. doi:10.1016/S0003-4975(03)00888-9

[12] N. Al-Sarraf, R. Aziz, K. Gately, J. Lucey, L. Wilson, E.
McGovern, et al., "Pattern and Predictors of Occult Mediastinal Lymph Node Involvement in Non-Small Cell Lung Cancer Patients with Negative Mediastinal Uptake on Positron Emission Tomography," European Journal Cardio-Thoracic Surgery, Vol. 33, No. 1, 2008, pp. 104 109. doi:10.1016/j.ejcts.2007.09.026

[13] W. Yang, Z. Fu, J. Yu, S. Yuan, B. Zhang, D. Li, et al., "Value of PET/CT versus Enhanced CT for Locoregional Lymph Nodes in Non-Small Cell Lung Cancer," Lung Cancer, Vol. 61, No. 1, 2008, pp. 35-43. doi:10.1016/j.lungcan.2007.11.007

[14] H. Nomori, K. Watanabe, T. Ohtsuka, T. Naruke, K. Suemasu and K. Uno, "The Size of Metastatic Foci and Lymph Nodes Yielding False-Negative and False-Positive Lymph Node Staging with Positron Emission Tomography in Patients with Lung Cancer," The Journal of Thoracic and Cardiovascular Surgery, Vol. 128, No. 3, 2004 pp. 396-401. doi:10.1016/i.jtcvs.2004.03.020 